\title{
Specificity in the Optical Excitation Lock-in Thermography Results Processing
}

\author{
Anna Stoynova ${ }^{1}$, and Borislav Bonev ${ }^{1, *}$ \\ ${ }^{1}$ Technical University of Sofia, Department of Microelectronics, Sofia, Bulgaria
}

\begin{abstract}
The aim of the paper is to study some factors influence on the contrast decreasing of the ampligram and phasegram obtained after optical lock-in thermography. It is analysed the effect of delay between excitation source modulated parameter changing and modulated signal due to thermal inertia of halogen lamp filament. Two software corrections for decreasing the effect of these factors are developed. In order to evaluate the effect of these corrections, lock-in thermography measurement is performed and multiple results processing at different correction parameters values are performed. The contrast dependence from correction parameters is presented in graphical form. Multiple results processing with random correction parameters values using the both corrections are performed for determination of maximum possible contrast An iterative method with less number of results processing for choosing appropriate values of correction parameters using the both corrections is proposed. The effect of this method is presented and compared in tabular and graphic form.
\end{abstract}

\section{Introduction}

In recent years, there has been a growing interest in active thermography methods, particularly lock-in thermography. Active thermographic methods use a forced temperature change (heating or cooling) of the tested object and simultaneously capture the process of temperature change with an infrared (IR) camera [1]. Excitation source is used for temperature change. In lock-in thermography is used a periodic temperature change.

The advantages of the lock-in thermography such as its non-destructivity and contactlessness in some cases, noise suppression and other, are a precondition for its wide application [2].

Any source of heating or cooling that can give or take heat for a long time can be used as excitation source:

- optical excitation sources (halogen lamp, LED, laser);

- mechanical excitation source (ultrasound source);

- electrical (for example - modulating the power supply voltage);

- hot air source.

The halogen lamp is one of the most commonly used optical excitation sources for lock-in thermography mainly because of its relatively low cost, high output power and relatively easy modulation of the light intensity.

The use of a halogen lamp as excitation source requires some peculiarities in the processing of the received data relating to the thermal inertia of the lamp [3].
A significant disadvantage in the use of a halogen lamp as excitation source is the poor energy efficiency of the measurement process. The efficiency of the halogen lamp is relatively low, which, combined with the long duration of the measurement process, leads to significant electricity consumption.

The energy efficiency of the process can be improve by increasing the sensitivity of the method, i.e., obtaining the same contrast at a lower light intensity of the lamp. Sensitivity is decreasing due to a number of factors whose origin and effect requires thorough analysis and finding ways to reduce their effect on the obtained result.

The LED optical excitation sources can be used also for energy efficiency improvement in cases, where it is not needed high output power of excitation source.

If it is needed heating of very small objects or spot of the tested object it can be used laser excitation source.

\section{Principle of lock-in thermography}

The principle of the lock-in thermography is based on the principle of synchronous phase detection. The first variants of lock-in thermography used analogue processing of the received signal by synchronous phase detectors. It was used a system with a sensor for infrared radiation and a modulated laser beam. This pair of emitter-receiver scans test surface [1]. Obviously, this system has been very slow. When IR thermal cameras become cheaper, they are used instead of the one receiver and the whole tested object surface is captured at once.

\footnotetext{
* Corresponding author: bonev@ecad.tu-sofia.bg
} 
An important point in the principle of the lock-in thermography is the need to know each thermogram of the sequence at which instantaneous value of the excitation source modulated parameter is captured.

The simplest way to realize this is the synchronization between the modulating signal of the excitation source and capturing thermograms from the IR camera.

An ampligram and a phasegram, as well as some other images, are obtained as a result from the lock-in thermography measurement. These are obtained by processing all or part of the thermograms from sequence.

There are basically two methods for processing the sequence of thermograms - digital lock-in correlation and Fourier analysis.

The principle of digital lock-in correlation is shown on fig. 1 .

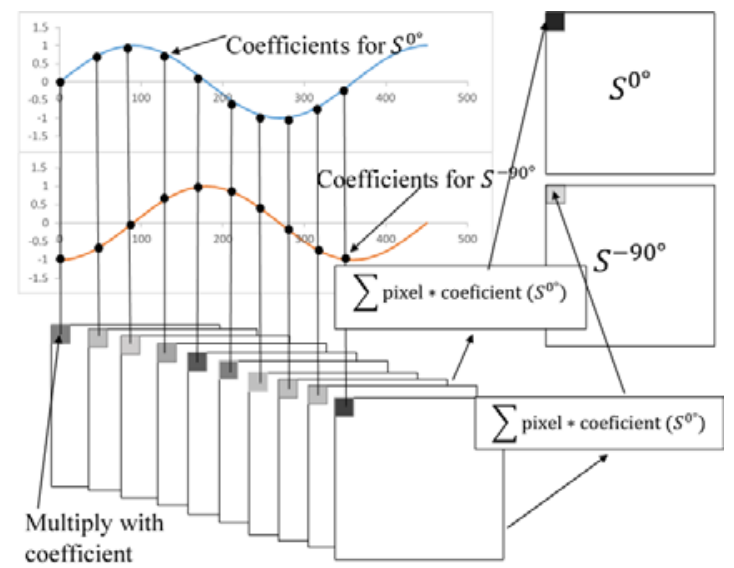

Fig. 1. The principle of digital lock-in correlation. [2]:

For one pixel of the images $\mathrm{S}^{0^{\circ}}$ and $\mathrm{S}^{-90^{\circ}}$ is obtained

$$
\begin{gathered}
S^{0^{\circ}}(x, y)=(1 / N n) \sum_{k=1: N}\left\{\sum_{m=1: n}\left[F_{k, m}(x, y) K^{0^{\circ}}\right]\right\} \\
S^{-90^{\circ}}(x, y)=(1 / N n) \sum_{i=k: N}\left\{\sum_{m=1: n}\left[F_{k, m}(x, y) K^{-90^{\circ}}\right]\right\} \\
K^{0^{\circ}}=2 \sin [2 \pi(m-1) / n] \\
K^{-90^{\circ}}=-2 \cos [2 \pi(m-1) / n]
\end{gathered}
$$

where:

$S^{0^{\circ}}(x, y)$ - value of the pixel $(x, y)$ from image $S^{0^{\circ}}$;

$S^{-90^{\circ}}(x, y)$ - value of the pixel $(x, y)$ from image $S^{-90^{\circ}}$;

$x$ - row of thermogram;

$y$ - column of thermogram;

$N$ - number of periods for which the calculation is performed;

$n$ - number of captured thermograms per period;

$F_{i, j}(x, y)$ - value of the pixel $(x, y)$ of image $i$ from period $j$

$K^{0^{\circ}}$ - correlation coefficient for computation of $S^{0^{\circ}}$;

$K^{-90^{\circ}}$ - correlation coefficient for computation of $S^{-90^{\circ}}$.

The ampligram and phasegram are obtained from images $S^{0^{\circ}}$ and $S^{-90^{\circ}}$ :

$$
S_{\text {amplidute }}=\sqrt{ }\left[S^{0^{\circ}}(x, y)^{2}+S^{-90^{\circ}}(x, y)^{2}\right]
$$

$$
S_{\text {phase }}=\operatorname{arctg}\left[S^{-90^{\circ}}(x, y) / S^{0^{\circ}}(x, y)\right]
$$

When it is used Fourier method for computation of ampligram and phasegram, first it is needed to calculate the first harmonic image:

$$
\begin{gathered}
S(x, y)=(1 / N n) \sum_{k=1: N}\left\{\sum_{m=1: n}\left[F_{k, m}(x, y) K\right]\right\} \\
K=\exp [-\mathrm{j}(2 \pi m / n]
\end{gathered}
$$

The ampligram and phasegram are obtained from the first harmonic image:

$$
\begin{gathered}
S_{\text {amplidute }}=\sqrt{ }\left[\operatorname{Re}(S(x, y))^{2}+\operatorname{Im}(S(x, y))^{2}\right] \\
S_{\text {phase }}=\operatorname{arctg}[\operatorname{Im}(S(x, y)) / \operatorname{Re}(S(x, y))]
\end{gathered}
$$

\section{Specificity in the use of an optical excitation source}

There are lot of specificities in thermogram sequence processing when it is used optical excitation source.

The direction of the voltage supplied to the halogen lamp is irrelevant. If it is supplied reverse voltage to the LED, it is off and can be damaged. Therefore, only one polar voltage is applied to halogen lamp and when it is used LED - only forward voltage. In the used experimental setup, halogen lamp voltage has the time diagram, shown on fig. 2.

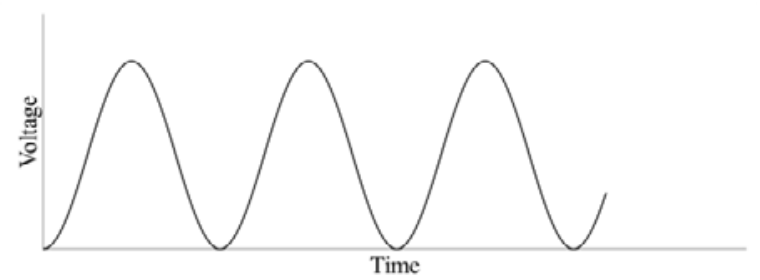

Fig. 2. The voltage waveform applied to halogen lamp.

When it is used digital lock-in correlation method, the closest correlation function to this signal is $(1-\cos )$ for computation of image $S^{0^{\circ}}$ and (1-sin) for computation of image $S^{-90^{\circ}}$.

As mentioned above, it is necessary to know the instantaneous value of the heat source modulated parameter for each thermogram of the sequence capturing time. The instantaneous value of the modulation signal is known for each image of the sequence capturing time. In the halogen lamp, the change in the light intensity does not fully follow the modulation signal, but with some delay due to the thermal inertia of the lamp filament. A solution in this case is the use of a light detector and the synchronization being the signal of the detector. However, software compensation may also be used. It changes initial delay of correlation function.

This delay, however, changes over time - at the start time, when the filament is cold, this delay is greatest. In subsequent periods, this delay is decreasing. This can be compensated by changing the frequency of the correlation function in combination with the initial delay.

Equations (3) and (4) are changed as follows: 


$$
\begin{gathered}
K^{0^{\circ}}=1-\cos \left[2 \pi(100+\mathrm{d})(m-1) /(100 n)-2 \pi t_{\text {delay }} f_{\text {lock-in }}\right] \\
K^{-90^{\circ}}=1-\sin \left[2 \pi(100+\mathrm{d})(m-1) /(100 n)-2 \pi t_{\text {delay }} f_{\text {lock-in }}\right]
\end{gathered}
$$

where:

$t_{\text {delay }}$ - delay time;

$f_{\text {lock-in }}$ - excitation source modulation frequency;

$\mathrm{d}$ - percentage change in frequency.

This choosing of values of delay and percentage frequency change depends not only on halogen lamp parameters, but also from many other factors environment parameters, tested object thermal parameters and other.

Automated determination of the most appropriate values for delay and percentage change of frequency can be realized. An indication that the most appropriate values for delay and percentage change of frequency are chosen is to achieve maximum contrast between two points or areas of the ampligram or phasegram. This includes multiple results processing and contrast comparison.

This can be applied in the case that in the results has a contrast, albeit low between the areas of the image. In this case, by varying the delay and frequency deviations, the contrast of the image can be increased.

When it is used Fourier method can also be used percentage frequency change correction. Equation (8) is changed as follow:

$$
K=\exp \{-\mathrm{j}[2 \pi m(100+d) /(100 n)]\}
$$

In the case of using a LED excitation source there are no delay between modulation signal and the change of light intensity due to low on/off time of LEDs, but the tested object thermal parameters and environment parameters affect and applying of corrections can be increase the contrast.

\section{Experiment and results}

To study the effect of the factors described above, a lock-in thermography measurement of a one-layer printed circuit board (PCB) was performed.

The halogen lamp and the camera are located opposite the PCB side without paths.

Characteristics of $\mathrm{PCB}$ :

- material - FR4;

- thickness - $1 \mathrm{~mm}$;

- material of PCB paths - copper;

- thickness of PCB paths $-35 \mu \mathrm{m}$.

Approximately calculation of the lock-in thermography measurement parameter is performed by using software Automation Technology IrNDT. A frequency of $0.2 \mathrm{~Hz}$ is chosen. At this frequency the heat wave penetration is approximately the thickness of the board [4].

The lock-in measurement is controlled by IRX-Box hardware and IR-NDT software of Automation Technology. The thermogram sequence is processed by own developed software in the environment of Matlab. It is used equations (1), (2), (11), (12), (5) and (6) for digital lock-in correlation method and (7), (13), (9) and (10) for Fourier method.

The used IR camera is FLIR SC640 with resolution of $640 \times 480$ pixels and frame rate of 30 frames per second.

The parameters of measurement are:

- lock-in frequency $-0.2 \mathrm{~Hz}(5 \mathrm{~s}$ period);

- measurement duration - 50s (10 lock-in periods);

- frame rate of IR camera - 10 frames per second.

It is not used all lock-in periods for computation of ampligram and phasegram, because the thermal process during the first few periods is far from thermal equilibrium and this leads to decreasing of contrast.

To determine the influence of the described error sources, a comparison of the contrast between two areas of the phasegram at different values of the percentage frequency change and delay time is performed. The areas used for contrast computation are shown on fig. 3 .

The contrast is calculated using equation (14).

$$
\begin{aligned}
\text { Contrast } & =\left(S_{\text {phase }}\left(a_{1}\right)+S_{\text {phase }}\left(a_{2}\right)+\ldots+S_{\text {phase }}\left(a_{10}\right)\right) / 10- \\
& \left(S_{\text {phase }}\left(b_{1}\right)+S_{\text {phase }}\left(b_{2}\right)+\ldots+S_{\text {phase }}\left(b_{10}\right)\right) / 10
\end{aligned}
$$

where:

$a_{1}, a_{2}, \ldots, a_{10}-$ pixel of area (a);

$b_{1}, b_{2}, \ldots, b_{10}-$ pixel of area (b). noise.

The averaging of 10 pixels is used for decreasing of

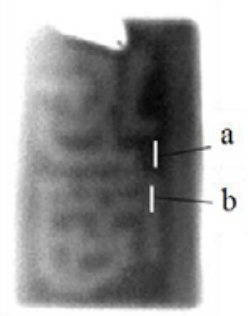

Fig. 3. Areas used for contrast computation.

The dependence between contrast and delay time is shown on fig. 4 . It can be seen that the contrast depends on the phase angle of the correlation function, as the output voltage in the synchronous phase detector depends on the phase angle between the reference signal and the frequency component of the input signal, the frequency of which is equal to the frequency of the reference signal. It can be seen that the maximum is not at zero delay, due to the delay between the change of the lamp light intensity and the modulation signal.

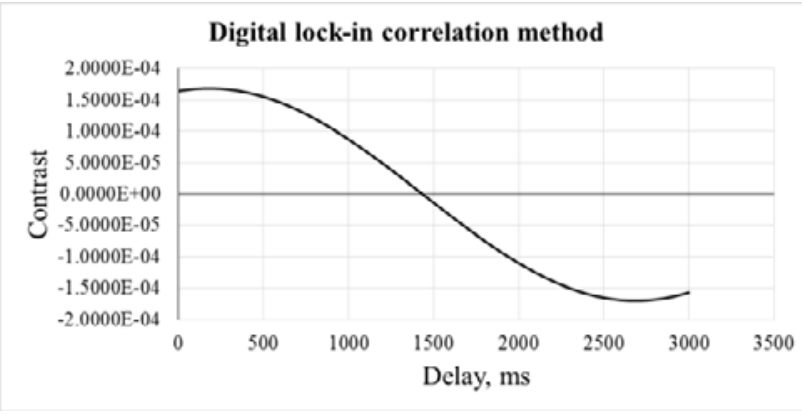

Fig. 4. Dependence between contrast and delay. 
The dependences between contrast and percentage change in frequency are shown on fig. 5. It is seen that the maximum is not at the frequency of the modulation signal. This is due to the variations in the delay between changing the light intensity of the lamp and the modulation signal.

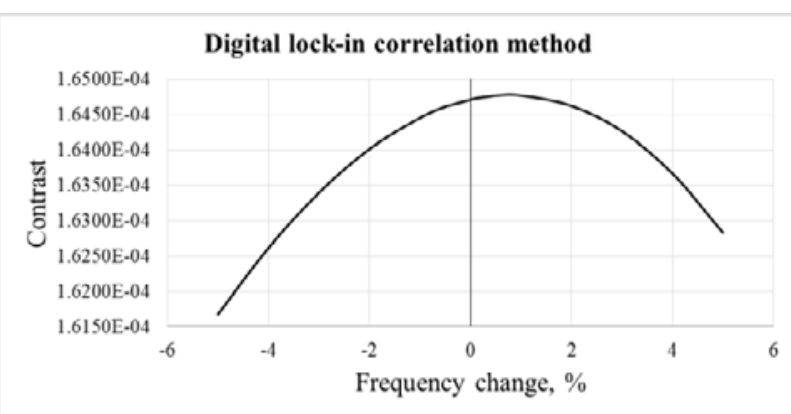

a)

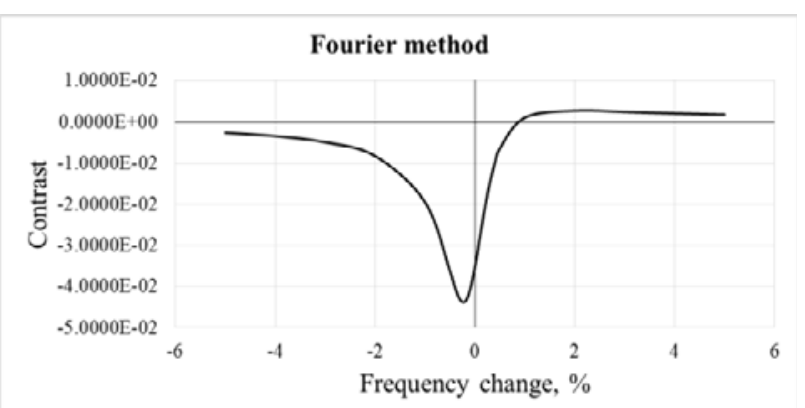

b)

Fig. 5. Dependence between contrast and percentage frequency change (a - digital lock-in correlation method, b - Fourier method).

The combination of the both corrections (delay and percentage change of frequency) can increase the contrast of phasegram more than if it is used one correction.

To determine the effect of the both corrections on the contrast, 50 phasegram calculations were performed using the digital lock-in correlation method at different random combinations of the delay and percentage frequency change values. A highest contrast is obtained at delay of $600 \mathrm{~ms}$ and percentage frequency change of $19 \%-1.7999 .10^{-4}$. Since the contrast depends on many factors such as lamp parameters, thermal parameters of the tested object, etc., the definition of dependence on which the values of delay and percentage frequency change can be calculated is difficult and the accuracy will be low. In this case can be used iterative method, whose block algorithm is shown on fig. 6 . The obtained values in this method are $200 \mathrm{~ms}$ for delay and $7 \%$ for percentage frequency change (the step for delay is $50 \mathrm{~ms}$ and for percentage frequency change $-0.5 \%$ ) after 18 computations of the phasegram and the obtained contrast is $1.7362 .10^{-4}$. The contrast without correction is $1.6472 .10^{-4}$. The comparison of obtained contrast at different values of delay and percentage frequency change is presented in table 1 .

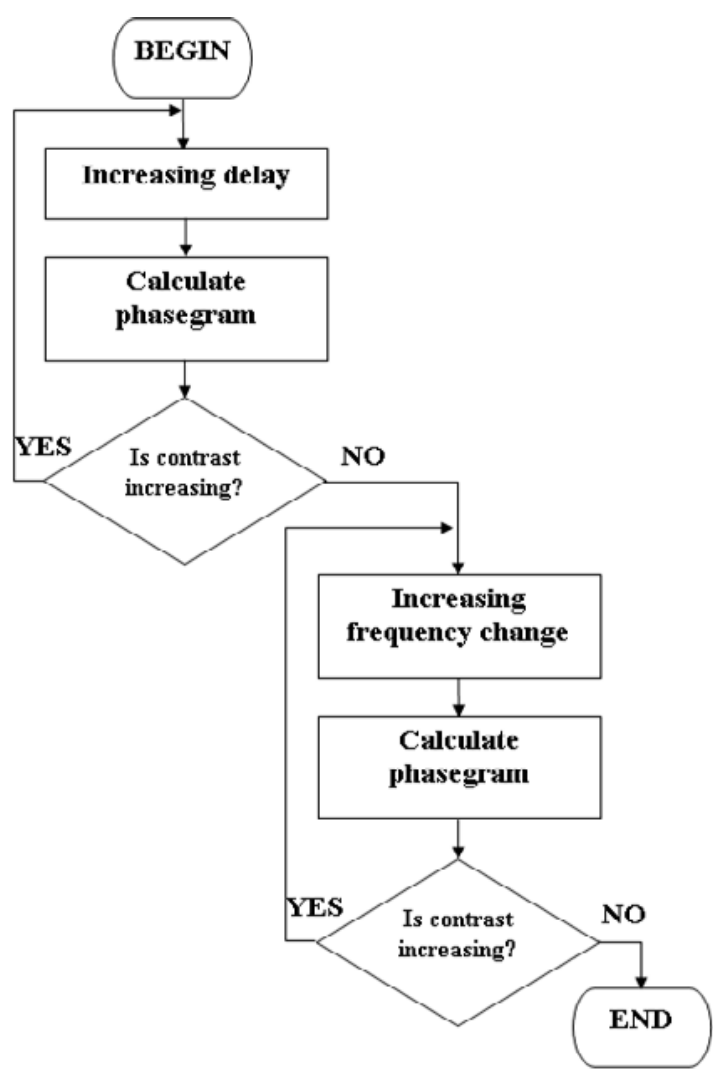

Fig. 6. Block algorithm of method for contrast increasing.

Table 1. Comparison between corrections.

\begin{tabular}{|c|c|c|}
\hline Correction & $\begin{array}{c}\text { Number of } \\
\text { computations }\end{array}$ & Contrast \\
\hline Without & 1 & $1.6472 .10^{-4}$ \\
\hline $\begin{array}{c}200 \mathrm{~ms} \\
7 \%\end{array}$ & 18 & $1.7362 .10^{-4}$ \\
\hline $\begin{array}{c}600 \mathrm{~ms} \\
19 \%\end{array}$ & $50^{*}$ & $1.7999 .10^{-4}$ \\
\hline
\end{tabular}

* This is a number of computations at random values of parameters. At other values it can be needed more computations for obtain the same contrast.

The phasegrams before and after using this method are shown on fig. 7 .
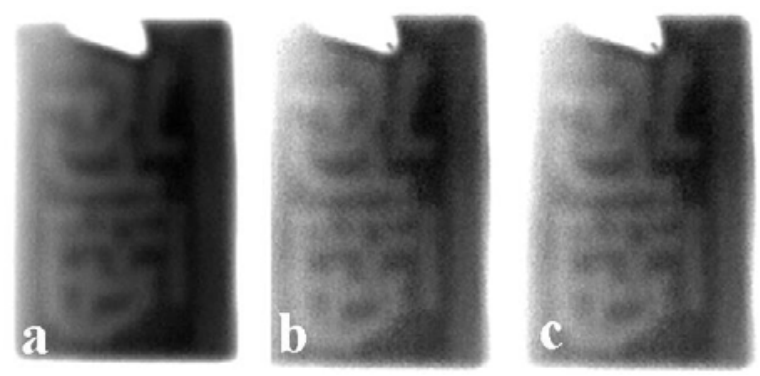

Fig. 7. Phasegrams before (a) and after using method for contrast increasing $(b-200 \mathrm{~ms}, 7 \%$ and $c-600 \mathrm{~ms}, 19 \%)$. 
The phasegrams before and after using percentage frequency change correction at Fourier method using equation are shown on fig. 8. The contrast without percentage frequency change correction is $-3.5213 .10^{-2}$ and with correction - $-4.3634 .10^{-2}$ at percentage frequency change $0.2 \%$.
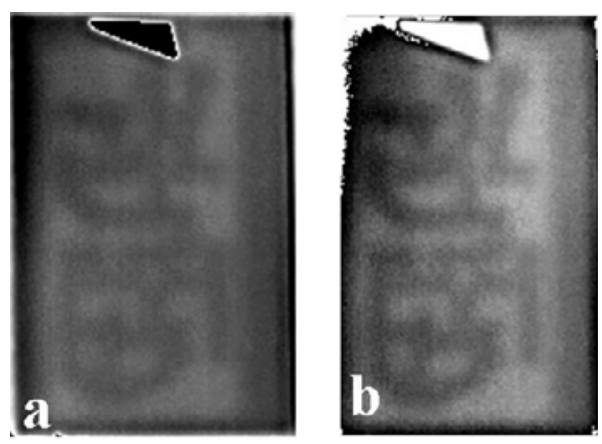

Fig. 8. Phasegrams before (a) and after (b) using percentage frequency change method at Fourier method.

\section{Conclusion}

The contrast of the images obtained after the lock-in measurement results processing is increased by using software compensation of the delay between the light intensity change of the lamp and the modulation signal in combination with a percentage change in frequency.

To obtain the same contrast as when no compensation is used, a lower lamp power is used which increases the energy efficiency of the method, as well as prolongs the life of the lamp.

\section{Acknowledgment}

The authors would like to thank for the support of Scientific Investigation Found under which Project No. BG05M2OP001-2.009-0033, the present work was conducted.

\section{References}

1. X. Maldague, Chapter XIV of the book: "Ultrasonic and Advanced Methods for Nondestructive Testing and Material Characterization", ed. Chen $\mathrm{CH}$, (2007)

2. O. Breitenstein, W. Warta, M. Langenkamp, Lock-in Thermography: Basics and Use for Evaluating Electronic Devices and Materials (Springer, New York, 2010)

3. G. Busse,, QIRT (2014), http://www.ndt.net/article/qirt2014/papers/QIRT2014-E.pdf

4. S. Delanthabettu, M. Menaka, B. Venkatraman, B. Raj, Quantitative InfraRed Thermography Journal, 12, 1, (2015) 